\title{
Une analyse non-substantialiste de l'architecture et du paysage
}

\section{A non-substantialist analysis of architecture and landscape}

\author{
Louis Vitalis ${ }^{1}$, Franck Li ${ }^{2}$, Malvina Apostolou ${ }^{3}$ François Guéna ${ }^{4}$ \\ ${ }^{1}$ Docteur en architecture, enseignant-chercheur au laboratoire de Modélisations pour l'Assistance à l'Activité \\ Cognitive de Conception (MAACC-MAP) à l'ENSA de Paris-La-Villette \\ ${ }^{2}$ Doctorant au laboratoire MAACC-MAP à l'ENSA de Paris-La-Villette \\ ${ }^{3}$ Doctorante au laboratoire MAACC-MAP à I'ENSA de Paris-La-Villette \\ ${ }^{4}$ Professeur à l'ENSA de Paris-La-Villette et Directeur du laboratoire MAACC-MAP
}

RÉSUMÉ. Un paysage dès lors qu'il est aménagé soulève la question de sa conception. L'architecture est également concernée par la conception spatiale, théorisée par l'architecturologie. Ces recherches peuvent-elles profiter aux études du paysage ? Cet article vise à tester l'opportunité d'un croisement entre ces deux arts : architecture et paysage. Le but est de permettre une étude scientifique des processus créatifs, qui analyse les caractères communs en se détachant des évidences substantielles. Afin d'y parvenir, l'idée que le paysage puisse entrer dans le champ de l'architecturologie est développée dans un premier temps. Un travail effectif d'interprétation architecturologique s'ensuit dans un deuxième temps, à travers trois situations paysagères : le cas du concours du parc de La Villette de 1982, le jardin chinois traditionnel de Suzhou et le nouveau quartier de Clichy-Batignolles organisé autour du parc Martin Luther-King. Enfin, après avoir repéré des similitudes et différences témoignant de cet apport, un croisement avec la mésologie d'Augustin Berque est réalisé dans le but d'enrichir la compréhension des différences entre ces conceptions paysagères dans leur rapport avec leurs milieux respectifs. Ainsi, l'architecturologie offre une description abstraite de la conception paysagère sans pour autant en exclure d'autres.

ABSTRACT. Landscape as a planning activity, raises the question of its design process. Spatial design concerns also architecture, and has moreover been studied by architecturology, a french design theory. Can this research benefit landscape studies? This article aims to test the relevance of a crossover between these two arts: architecture and landscape. The purpose is to enable a scientific study of creative processes that analyses the common features detached from the substantial evidence. First, we will explore the applicability of the architecturological theory to other, non-architectural objects. Second, we pursue an empirical architecturological interpretation of three landscape situations: the competition for the park of La Villette in 1982, the traditional Chinese garden of Suzhou and the ClichyBatignolles district organised around the Martin Luther-King park. This work allows to identify similarities and differences between these landscape designs and thus supports the relevance of a landscape architecturology. Finally, to better understand the role of cultural context in design, a crossover with Augustin Berque's mesology is carried out. Thus, architecturology enables an abstract analysis of landscape design among others possible.

MOTS-CLÉS. conception, architecturologie, mésologie, échelle, parc, jardin, paysage.

KEYWORDS. design, architecturology, mesology, scale, park, garden, landscape.

\section{Introduction}

« Ni en architecture, ni en géographie, en principe on ne peut faire abstraction du monde sensible et de l'existence humaine, qui en est la mesure. Non seulement l'on ne peut pas en faire abstraction mais c'est l'étude ou l'aménagement de cette échelle même - de ce rapport entre l'humain et le terrestre — qui motive et la géographie et l'architecture ! ${ }^{1}$

Cette remarque d'Augustin Berque nous intéresse parce qu'elle met sur le même plan géographie et architecture. Il suggère dans son livre que la question de l'échelle est importante pour se saisir de la question du paysage, mais qu'elle est aussi affaire d'« aménagement ». C'est-à-dire non pas d'un

\footnotetext{
${ }^{1}$ Berque, 2010, 114-15

C 2020 ISTE OpenScience - Published by ISTE Ltd. London, UK - openscience.fr
} 
paysage déjà là, donné, mais conçu. Pour ces raisons il nous apparait qu'une théorie de l'échelle à même de décrire des processus de conception serait utile à la recherche en paysage. Or il existe une telle théorisation: l'architecturologie ${ }^{2}$. En se focalisant sur le processus de conception, elle se détache d'une analyse de l'architecture comme substance réalisée. Mais elle porte apparemment sur l'architecture. Est-ce un obstacle ? Voilà la question que pose cet article.

Deux mots sur l'architecturologie : l'échelle y est définie comme une opération d'attribution de mesure. Le réel de l'architecte n'est pas donné, mais construit. Il ne saurait prendre les mesures de son architecture puisque celle-ci n'existe pas encore. Il lui faut d'abord les lui donner. Mais il y a avant tout une pluralité d'échelles qui résulte des différentes modalités d'attribuer des mesures à l'espace. Les échelles opèrent selon des référents extérieurs qui jouent le rôle de «mesurant» permettant d'attribuer des mesures à des dimensions, soit des entités de l'espace mentalement découpées. En fonction des références (vue, usage, structure porteuse...) des classes d'échelles sont définies (échelle de visibilité, fonctionnelle, technique...). La conception est alors architecturologiquement décrite comme le jeu complexe de relation entre différentes échelles. Pour situer l'ambition de ce travail théorique, il faut comprendre qu'il vise une certaine généralité. Chaque projet d'architecte est singulier c'est entendu. Mais l'architecturologie s'attache à déceler ce qui peut être commun dans les manières d'opérer des concepteurs. Il s'agit in fine d'un langage conceptuel permettant d'enseigner la conception ou encore de comparer des conceptions malgré les distances historiques et culturelles ${ }^{3}$.

Ce langage peut-il alors déceler quelque chose de commun entre le paysage et l'architecture indépendamment de leur substance ? Si l'idée d'une architecturologie du paysage a pu être aperçue $^{4}$, nous chercherons ici à la développer. Nous établirons, dans un premier temps, pourquoi l'on peut considérer l'architecturologie de jure utile pour parler du paysage. Dans un second temps, l'on envisagera comment de facto cela peut avoir lieu.

\section{Architecturologie - au-delà de l'architecture}

\section{L'interprétation axiomatique}

Il nous faudra d'abord clarifier la situation discursive en posant la question suivante : pourquoi l'architecturologie ne concernerait que l'architecture ? Il s'agit de revenir sur l'évidence verbale de cette association, sur ce qui semble acquis : la raison principale de l'association architecturearchitecturologie, repose sur le terme. L'étymologie «architecturo-logie » indique la présence de l'architecture. Mais cette présence est-elle uniquement étymologique?

Si l'on pose la question de savoir pourquoi l'architecturologie se nomme architecturo-logie, on s'interroge finalement sur ce que l'architecturologie est susceptible de nommer architecture. Il semble que ce soit un problème de définition (qu'est-ce qu'on appelle architecture en architecturologie ?) que l'on voudrait toutefois distinguer d'une question essentialiste du type

\footnotetext{
${ }^{2}$ Initiée par Philippe Boudon aux débuts des années 1970, elle est le fruit d'un collectif de chercheurs des ENSA de Nancy et de Paris-La Villette.

3 Pour compléter cette brève présentation, se référer au manuel (Boudon, Deshayes, Pousin, \& Schatz, 1994/2000) ou à l'ouvrage introductif (Boudon, 1992).

${ }^{4}$ Catherine Chomarat-Ruiz s'est penchée sur l'architecturologie dans le but de construire sa « paysagétique ». Elle conclut qu' « il n'existe pas une approche des sciences de la conception qui [...] pourrait être transposée au paysage de façon satisfaisante » (Chomarat-Ruiz, 2014, p. 199). Philippe Boudon pour sa part considère que l'interprétation de Catherine Chomarat-Ruiz procède $d^{\prime}$ ' une fausse interprétation de l'échelle " (Boudon, 2015). Pour sortir de cette impasse, cet article veut proposer de reconsidérer la question.
} 
«qu'est-ce que l'architecture?» (question à laquelle on n'a jamais fini de répondre, parce que n'ayant pas de cadre).

Pour comprendre ce que l'architecturologie sous-entend par architecture, il faut rappeler sa question scientifique principale, celle d'expliciter comment l'architecte donne des mesures à l'espace. Car le système conceptuel qu'elle a construit pour éclairer cette question donne en creux une idée de ce qu'elle entend par architecture. Cette question repose sur la conjonction de deux postulats fondamentaux : toute architecture est conçue et toute architecture est mesurée.

S'il y a architecture donc, ce n'est que par ces deux aspects ; «l'architecte », du point de vue architecturologique, n'est architecte que parce qu'il donne des mesures et n'importe quel individu en ce sens est architecte dès lors qu'il donne des mesures, quels que soient son titre ou sa formation (l'architecturologie ne vise pas une connaissance sociohistorique, à chaque théorie son objet).

L'architecturologie n'aurait-elle alors pas gagné à s'appeler spatiologie, designologie... ? face à cette question de mots, un anti-essentialisme poppérien fournit une issue thérapeutique : les querelles de mots sont sans importance, ce qui compte ce sont les faits. Peut-être alors qu'architecturologie est un très mauvais mot, peu importe.

Le problème qui nous importe est alors curieusement similaire à celui qui s'est posé dans la réflexion sur les axiomatiques, ces constructions logiques qui cherchaient à représenter la structure de théories pour notamment évaluer leur cohérence indépendamment de leur contenu substantiel. Comme l'indique le logicien Robert Blanché, la question est de se détacher d'intuitions naturelles pour se limiter à ce qui est explicitement énoncé dans la structure d'une théorie. Il s'agit donc de ne pas faire dire plus à la théorie que ce qu'elle contient ou que la combinaison de ses éléments permet d'énoncer. Ainsi la logique raisonne sur des propositions abstraites, symbolisées par des lettres, pour évacuer un sens vague véhiculé par les mots d'usage courant. Le sens est fixé par les postulats, " lesquels énoncent quelles relations logiques soutiennent entre elles ces notions » (Blanché [1955] 1990, 37-38).

Ces relations logiques vont alors structurer ce que Blanché appelle des définitions implicites. Cette notion nous permet alors d'échapper au problème qui concerne la définition et le sens, en les faisant reposer non sur ce que ces mots signifieraient en eux-même, mais sur ce qu'un système conceptuel définit de manière implicite. Il sera alors fécond de suivre cette perspective axiomatique et en particulier l'idée suivante : "Considérons maintenant une seule de ces multiples axiomatiques d'une théorie concrète. Puisque le sens de ses termes, et par conséquent de toutes ses propositions, n'est fixé par les postulats que de façon équivoque, on pourra toujours, si l'on trouve plusieurs systèmes de valeurs qui satisfont également à l'ensemble des relations énoncées par les postulats, en donner des interprétations concrètes diverses ou, autrement dit, choisir entre plusieurs réalisations. Ces réalisations concrètes d'une axiomatique sont appelées ses modèles » (Blanché [1955] 1990, 4547). Il y a donc trois niveaux qui peuvent être schématisés selon la Figure 1 :

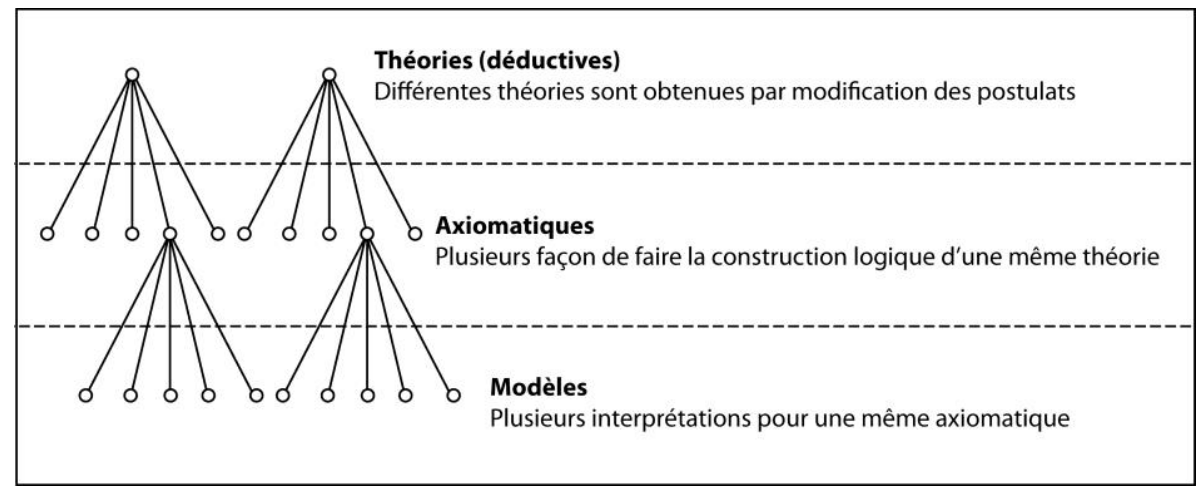

Figure 1. Les trois niveaux de la réflexion axiomatique (schéma L. Vitalis d'après Blanché, 1955/1990) 
Si l'on applique à l'architecturologie cette distinction en se limitant ici au rapport de la théorie à ses interprétations ${ }^{5}$, la situation est alors décrite par la Figure 2 :

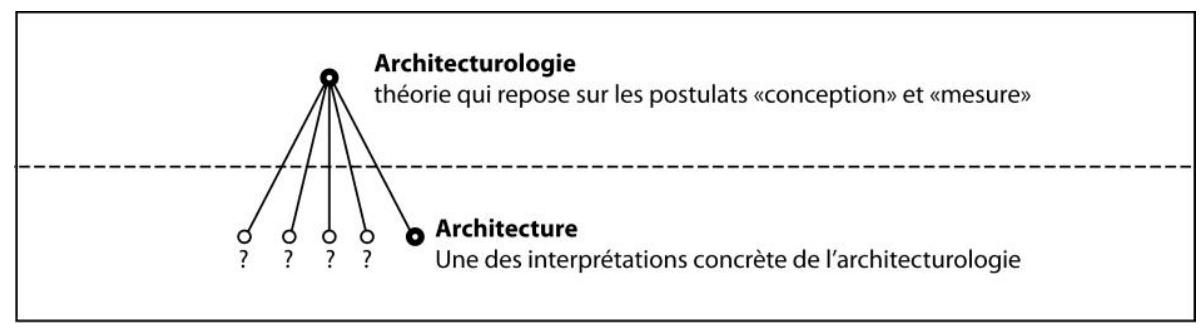

Figure 2. Le rapport architecturologie/architecture en tant que rapport théorie/interprétation (schéma L. Vitalis)

Ce qui importe ici est l'idée que l'architecture n'est qu'une des interprétations concrètes de l'architecturologie (un de ses modèles). Dès lors, d'autres modèles sont possibles et il existe, en droit, une multitude d'interprétations possibles de l'architecturologie. Et si l'on s'interroge alors sur les raisons pour lesquelles l'architecturologie pourrait intéresser d'autres domaines, il est possible d'arguer que, repartant des postulats, c'est le cas pour tout domaine concerné par la conception et la mesure. Voici deux de ces domaines :

\section{Architecturologie du Testament d'un excentrique de Jules Verne}

Ce roman de Jules Verne, qui raconte les péripéties de six héritiers potentiels à travers les USA, a pour sujet le pays lui-même en quelque sorte. En cela il pose une question de conception littéraire puisqu'il tente de faire entrer un immense territoire... en 250 pages. Lorsque l'auteur se pose cette question, il est face au problème de devoir transformer un espace à (au moins) deux dimensions, en une narration linéaire d'une dimension. La solution trouvée est celle d'un jeu de l'Oie, où chaque case est un état. Le notaire déplace les pions représentant les héritiers et leur télégraphie l'endroit où se rendre.

Ce choix de conception a pu être architecturologiquement décrit comme une échelle de modèle (Boudon 1988), le jeu de l'Oie étant pris comme modèle pour réutiliser sa structuration linéaire de l'espace dans celle des chapitres du livre. Le concepteur du roman par ce choix met également en place une échelle extension, car faisant jouer les coups de dés qu'il désire au notaire, il pourra agrandir à souhait le roman pour ajuster le nombre de pages. Cette structure faisant correspondre un coup de dés à un chapitre, et donc à une histoire dans l'histoire simplifie le travail de l'écrivain par une échelle économique qui permet de découper le travail d'écriture complexe en fragments simples.

\footnotetext{
${ }^{5}$ La question de la construction axiomatique formelle de l'architecturologie sera laissée de côté ici. Si une première base a été posée dans (Boudon, Decq, Deshayes, de Gandillac, \& Schatz, 1975, p. 72-91), les débats qu'elle appelle - du fait notamment de son inspiration wittgensteinienne et de l'état précoce de la théorie en 1975 - engageraient à des développements qui ne sont pas l'objet de cet article.
} 


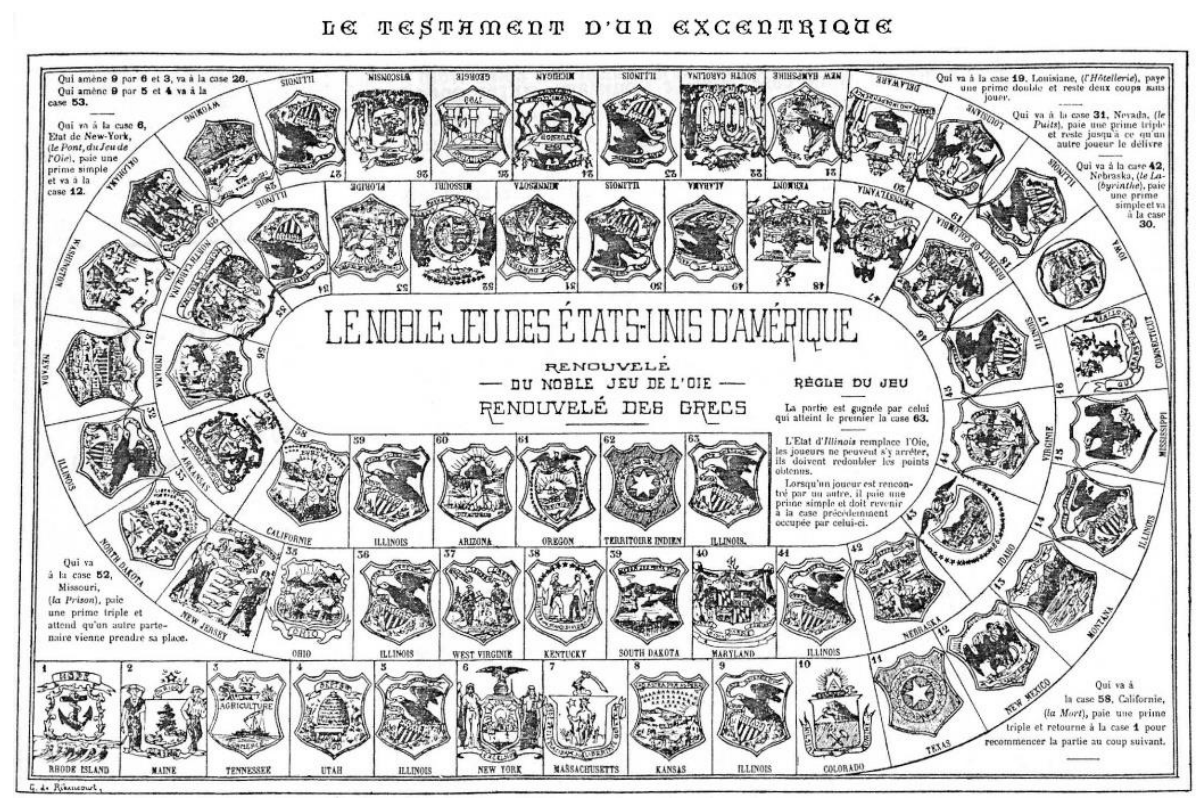

Figure 3. Le noble jeu des États-Unis d'Amérique (Jules Verne)

\section{Architecturologie du poème optique de Man Ray}

Cette œuvre de Man Ray se présente comme un texte où les mots et phrases ont été représentés par des traits qui marquent simplement la série prosodique des unités signifiantes en les vidant de tout signifié. En considérant ce « poème » comme la mise en œuvre d'une échelle optique (Boudon, 2004) l'architecturologie permet de décrire comment des dimensions - les mots du poème - sont mesurées en fonction de références, ici le rythme visuel, et pour une pertinence rythmique (accélération ralentissement...).

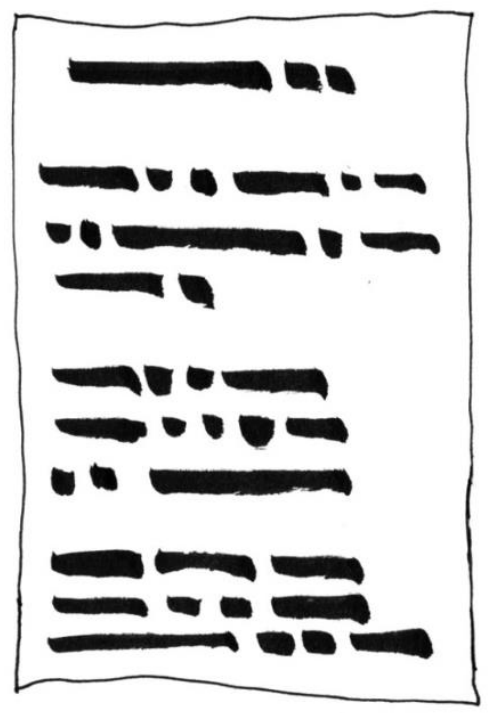

Figure 4. Poème optique à la manière de Man Ray (source : L. Vitalis)

Certes cette opération de conception ne suffira pas à la conception complète du poème (il manque des mots), mais de même que l'architecturologie ne prétend pas décrire l'architecture complètement, elle permet de se focaliser ici sur un aspect particulier de la poésie. L'architecturologie permet de décrire une phase précoce de la conception dans laquelle il s'agit d' " intentionner un poème avant même qu'il y ait poème » (Boudon 2004, 20). 
Dès lors d'autres exemples d'échelle optique pourront être trouvés dans le poème de Mallarmé, «Un coup de dés jamais n'abolira le hasard », qui, par les mesures qu'il attribue à la taille des mots, à leur distance et leur position sur la feuille, évoque visuellement le lancer de dé et l'apparition du hasard. À partir de cet exemple un travail systématique a proposé des hypothèses d'application de 14 échelles architecturologiques à des poèmes et romans divers (Boudon 2004, 21-22).

Ces deux cas indiquent qu'en plus de l'architecture, la littérature et la poésie sont des domaines d'interprétation possibles pour l'architecturologie. Si le paysage peut être considéré comme un objet artificiel spatial (artificiel au sens de Herbert A. Simon : qui est conçu), il semble être alors un domaine opportun pour l'architecturologie. Cela ne signifie alors aucunement de faire du paysage une architecture. L'architecturologie n'implique pas, selon nous, de substance architecturale.

\section{Quelques descriptions architecturologiques de projets de paysage}

Maintenant que la possibilité d'une architecturologie du paysage est établie en droit, nous envisagerons comment elle fonctionne empiriquement. Soit des situations paysagères différentes, éloignées historiquement et culturellement : comment pouvons-nous les comparer ? L'expérience qui est proposée est de produire une interprétation architecturologique de ces paysages. Le fait que les concepts de l'architecturologie permettent d'exprimer des différences et des similitudes de ces cas sera considéré comme signifiant un apport possible de l'architecturologie à l'analyse de la conception paysagère. Pour ce faire nous analyserons trois cas : le concours du parc de La Villette de 1982, le jardin chinois traditionnel de Suzhou et le nouveau quartier de Clichy-Batignolles organisé autour du parc Martin Luther-King.

\section{A. Parc de la Villette}

Le concours pour le parc de La Villette de 1982 proposait aux concepteurs de réfléchir à un parc urbain de 55 ha. À l'issue d'un premier tour du jury 9 prix ex-aequo sont décernés dont plus de la moitié des mandataires sont architectes : Van Gessel, Ariola, Gouvennec, Koolhaas et Tschumi, et presque autant de paysagistes : Vexlard, Anderson, Lassus et A. Chemetoff (EPPV 1982). Voyons maintenant trois extraits de leurs projets :

\section{Les haies du parc de Bernard Tschumi}

Focalisons-nous sur la couche dite des « lignes » dans la fameuse axonométrie éclatée pointslignes-surfaces du projet de Bernard Tschumi. Les lignes représentent du végétal ; pourtant les différentes représentations (cf. Figure 3) montrent des dessins très géométriques dont l'abstraction ne correspond pas à l'idée d'une nature libre. Cette façon de dessiner le végétal interroge sur la manière dont les concepteurs ont appréhendé le paysage.

Le fait que les haies sont dessinées comme des lignes ne peut être tout à fait fortuit, mais révèle une intention. L'architecturologie permet alors de décrire ce cas de conception comme un découpage général en trois niveaux de conception : points, lignes et surfaces. Ce découpage procède de la surdétermination d'une échelle sémantique (référence aux mots de Kandinsky dans son livre Point et ligne sur plan), d'une échelle géométrique (en référence à des qualités géométriques prédéfinies) et d'une échelle fonctionnelle (en référence à des usages : des promenades dans les lignes, des buvettes, kiosques et abris divers dans les points), des surfaces appropriables par des activités sportives par exemple. 

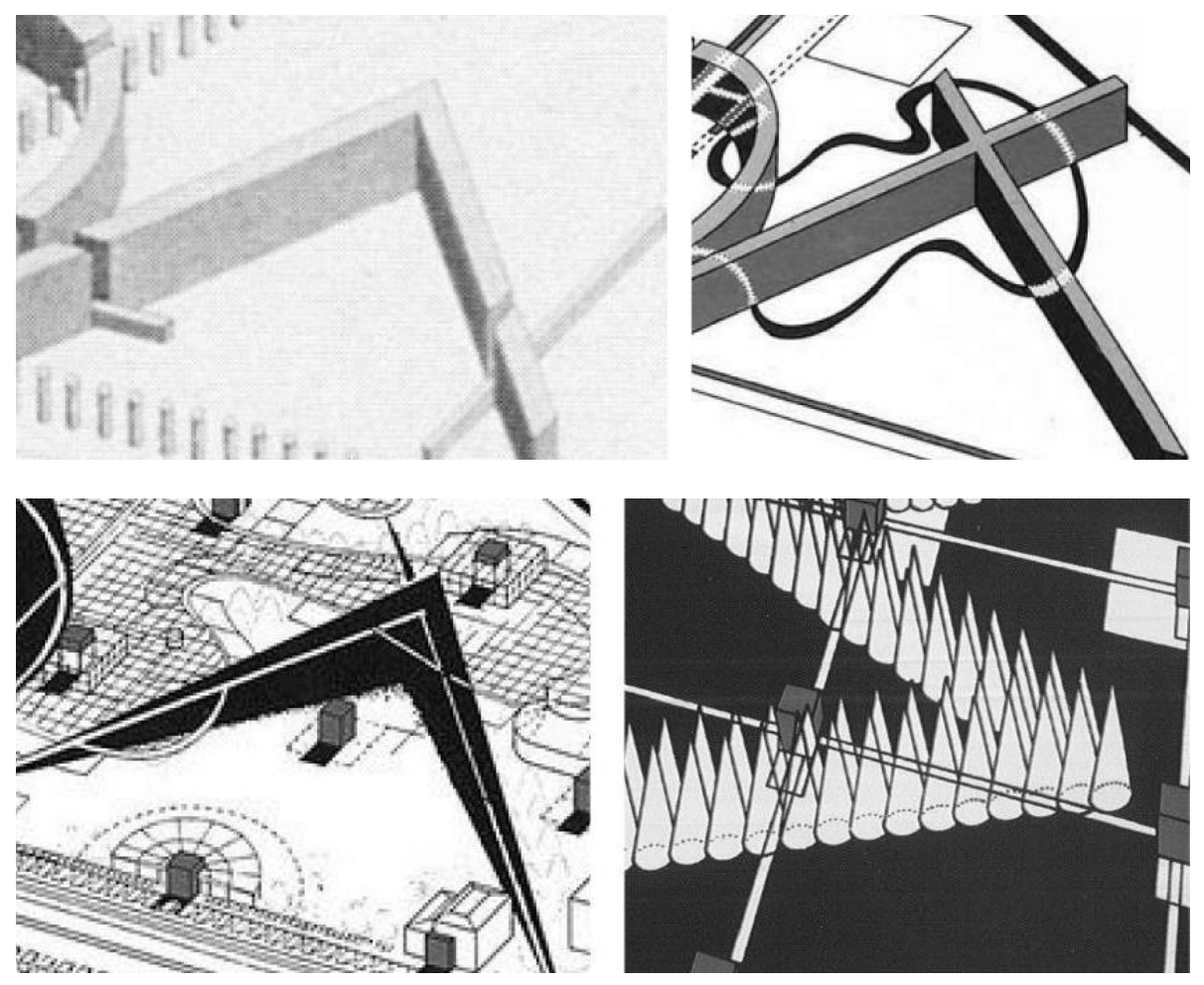

Figure 5. Extraits des différentes axonométries du projet de Bernard Tschumi (source : extraits de Bernard Tschumi Architects, montés à partir de (Tschumi 1982, 11), (Tschumi 2014, 43), (Tschumi 1989, 17, 7)).

Chaque niveau est ensuite conçu de manière relativement indépendante. Les lignes sont notamment à nouveau découpées selon une échelle fonctionnelle : traversée rapide, lente, ou promenade sinueuse. Enfin, la conjonction des trois niveaux a pour pertinence de produire des situations de rencontres inédites entre espaces et activités. L'idée de « patiner dans la serre tropicale au son du piano » (Tschumi 1984, 34) en est un exemple.

Le caractère géométrique du traitement du végétal trouve ainsi une explication au niveau de la conception : le découpage général du projet selon des références géométriques permet de traiter l'ensemble du programme (références fonctionnelles) malgré sa complexité en une suite d'opérations selon une pertinence globale : celle de rencontres inédites.

Certes, le fait que la description architecturologique fonctionne pour ce cas peut être lié au fait que les concepteurs approchent le paysage en architectes. Ainsi, l'architecte Luca Merlini, le collaborateur de Bernard Tschumi lors du concours, affirme lors d'un entretien : «Le programme n'était pas le programme d'un parc, mais c'était peut-être le programme d'un morceau de ville, [...] le végétal, n'était pas... était un matériau de construction, comme l'étaient d'autres matériaux de construction. C'est-à-dire que l'on pouvait faire des murs de végétaux comme on pouvait faire des murs de briques » (Merlini \& Vitalis, 2018)

\section{La forêt ronde du parc de Rem Koolhaas}

Pour prendre alors un cas où le végétal est traité pour sa spécificité et non comme analogue à un bâti, envisageons maintenant le projet du parc de Rem Koolhaas et en particulier, la forêt ronde. De manière similaire, le projet de Rem Koolhaas superpose quatre couches: les bandes programmatiques, les confettis, les circulations et les grands éléments dont fait partie la forêt ronde. Ces grands éléments sont censés contrebalancer la présence de grands éléments bâtis présents sur le site (la Grande Halle et la Cité des Sciences) par des éléments architecturaux.

L'intérêt principal de la forêt circulaire est qu'elle est conçue pour permettre au cours de sa croissance d'offrir une variété d'états dont tous offrent des qualités et non seulement les états de 
maturité des arbres. Pour cela, la forêt est constituée d'une alternance régulière de cyprès et de cèdres du Liban (Lucan 1990, 63).
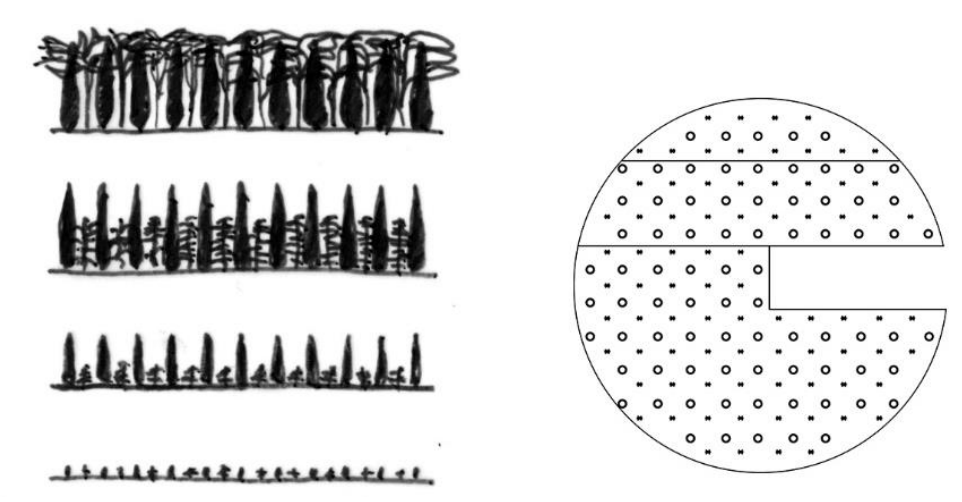

Figure 6. Les essences et la croissance de la forêt ronde (source : L. Vitalis, d'après-Koolhaas, 1990, 63)

D'un point de vue architecturologique, on retrouve ici l'échelle géométrique, qui fait jouer le rond comme forme pure évoquant une nature maîtrisée. Ce cas peut aussi être décrit comme un cas emblématique d'échelle d'extension. C'est-à-dire que plusieurs mesures sont attribuées ici : un découpage définit d'abord deux types d'essences à déterminer, essences qu'il faut ensuite disposer et espacer... et ce qui guide ces attributions de mesure, c'est la référence au devenir de la forêt. Différencier les essences est pertinent pour varier les qualités spatiales en jouant sur deux croissances végétales différenciées. Les cyprès offrent une expérience intéressante dès 1987, rappelant les jardins à la française, tandis que le développement horizontal plus tardif de la ramure des cèdres du Liban aboutit à une « majestueuse rangée de colonnes coiffés d'un toit vert sombre » (Koolhaas 1983, 98).

\section{La pente du parc de Bernard Lassus}

Pour en terminer avec le parc de La Villette, prenons la proposition d'un paysagiste, le projet de Bernard Lassus. Plus particulièrement, arrêtons-nous sur la grande « pente » avec laquelle il couvre la presque totalité du site.

Cette pente reprend la déclivité naturelle de la vallée de la Seine du site de La Villette, que les aménagements urbains ont effacée : «il nous semblait pertinent de reproduire, à l'aide d'un grand plan incliné, la pente initiale du site » dit Bernard Lassus (entretien in Orlandini, 1999/2003, p. 146). Cette idée de reproduction indique d'un point de vue architecturologique la présence d'un modèle et donc du point de vue des opérations de conception d'une échelle de modèle.

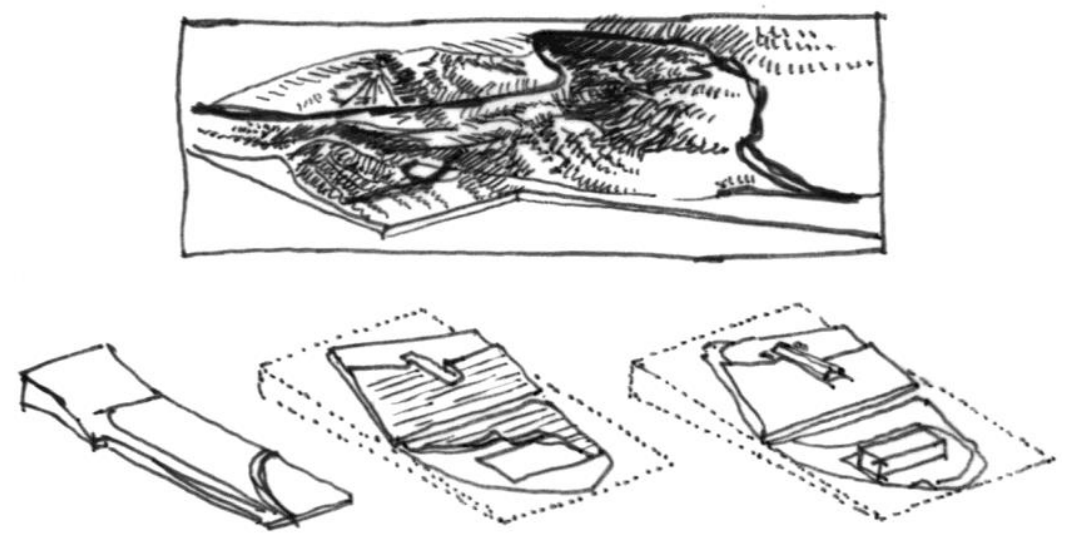

Figure 7. La vallée de la Seine mise à l'échelle du parc de La Villette (source : L. Vitalis, d'après Lassus, 1998, p. 123) 
La pente de la Vallée de la Seine sert de modèle à Bernard Lassus, qui travaille à la mettre à l'échelle du site de La Villette. La pertinence de ce modèle est de créer pour l'utilisateur une «perception géographique» selon les mots du concepteur. La pente ainsi reproduite sur le site « opère, comme une jonction, entre la porte de La Villette au nord et la porte de Pantin au sud et comme métaphore visuelle de la pente de Belleville »(Lassus 1983, 135). Cette dernière déclaration indique bien que la référence de l'opération de conception est la vue. Une échelle optique vient donc prendre le relai de l'échelle de modèle. En faisant disparaître une partie de la Grande Halle, la pente constitue visuellement un horizon. Cette situation optique est le moyen de produire la « perception géographique ». Notons que cette pente était artificielle et abritait en fait une quantité de programmes prévus pour le parc.

\section{B. Jardin de la Politique des Simples, Suzhou}

Le jardin de la Politique des Simples (ou jardin de l'Humble Administrateur) situé dans la ville de Suzhou, en Chine, a été conçu et construit à partir de 1509 bien que réaménagé à plusieurs reprises au cours de son histoire. Il s'agit d'un jardin bien conservé et documenté offrant de multiples scènes, éléments caractéristiques des jardins classiques chinois. Cette notion de scène peut être comprise comme un espace conçu, pour être perçu et interprété par le promeneur laissant alors «son esprit vagabonder ${ }^{6}$ au-delà du réel (Jie et Xin 2017, 336). Nous étudierons le regard que porte l'architecturologie sur la conception relative à la perception dans le cadre de ces scènes paysagères.

\section{Voir et être vu}

Intéressons-nous d'abord à l'aspect visuel de la perception à travers l'idée d'emprunt de scène, décrit dans le Yuanye $e^{7}$ comme un dispositif majeur des jardins. Il est au premier abord défini par le fait que point de vue et élément scénique soient associés. L'élément mis en scène peut être situé en dehors du jardin d'où le terme d'emprunt (Fung, 1999). En l'occurrence, dans le jardin du simple politicien, nous pouvons considérer que la scène vue depuis le Pavillon-Bambou, illustrée par la figure 8, emprunte la Pagode-du-nord, un objet de 1'espace extérieur au jardin. Cet emprunt de la pagode peut être compris architecturologiquement comme une échelle de visibilité au sens d'une «opération de conception par laquelle est constitué un point de vue » (Boudon 1983, 150). À Suzhou, le concepteur tient compte de la Pagode-du-nord pour créer un point de vue en positionnant le Pavillon-Bambou et son ouverture circulaire.

\footnotetext{
${ }^{6}$ Traduit de l'anglais « mind-wandering »

${ }^{7}$ Traité du jardin rédigé par Ji Cheng au $17^{\mathrm{e}}$ siècle, abordant la conception du jardin classique chinois 


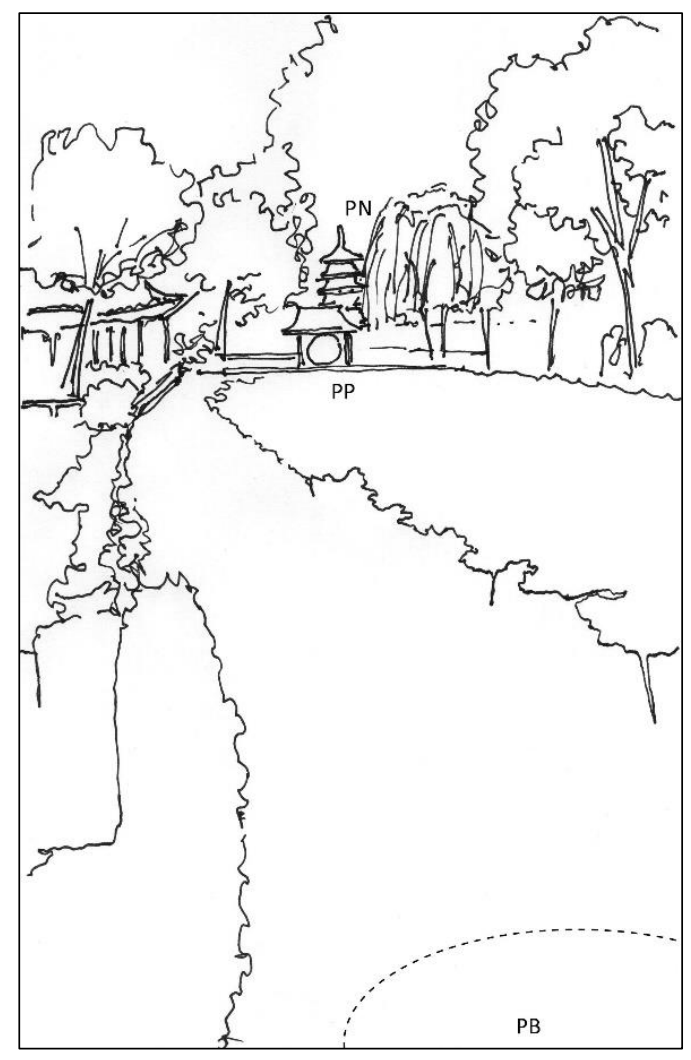

Figure 8. Esquisse de la scène empruntant la Pagode-du-nord (source : F. Li d'après Colson \& Sullivan, 2010, p. 188). PB : Pavillon-bambou, PN : Pagode-du-nord, PP : Porte-pavillon-d'un-pavillon-au-charmeexceptionnel

Sur la figure 8, une porte-pavillon se situe également dans l'axe de vision. Ainsi l'échelle visibilité a tenu de deux références. Cette porte-pavillon se trouve être également un point de vue sur le Pavillon-bambou. L'emprunt de scène fonctionne ici à double sens : le Pavillon-bambou est emprunté par la Porte-pavillon. Nous pouvons déceler la présence d'une échelle optique, soit « l'opération ayant pour objet ce qui est regardé » (Boudon 1983, 150).

Il est remarquable que cet emprunt de scène fasse intervenir deux points de vue en situation de réciprocité. Néanmoins, en distinguant échelle de visibilité et échelle optique, l'architecturologie indique qu'il y a dans la conception quatre situations distinctes : le positionnement du Pavillon bambou tient ici compte de deux points de vue, mais ceci est également vrai pour la porte-pavillon. Ces quatre opérations de conception peuvent être résumées dans la figure 9. Un même pavillon est alors positionné selon deux points de vue, celui qu'il offre et celui depuis lequel il est vu. L'architecturologie désigne cette relation de relance entre échelles comme une codétermination.

En outre, d'autres éléments (pavillons, belvédères, ouvertures) forment un jeu relationnel entre une multiplicité de points de vue. Cette complexité des points de vue peut être représentée par le diagramme de la figure 10. 

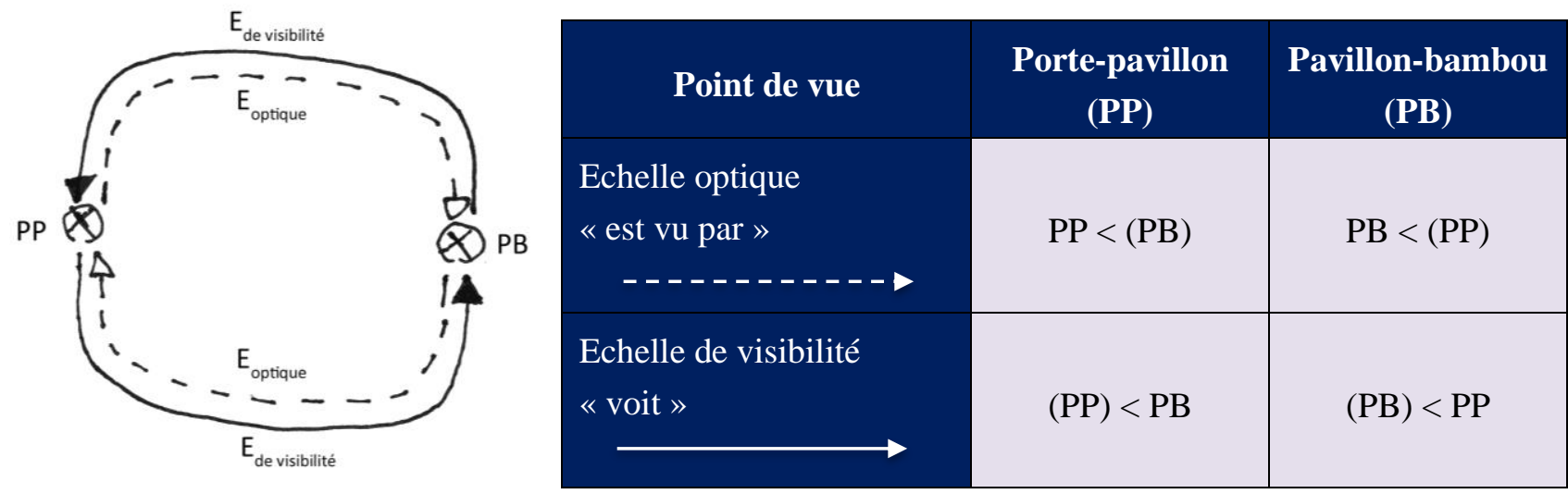

Figure 9. Schéma des quatre opérations de conception liées à la vue (source : F. Li) Tableau 1. Tableau associé à ces quatre opérations distinctes (source : F. Li)

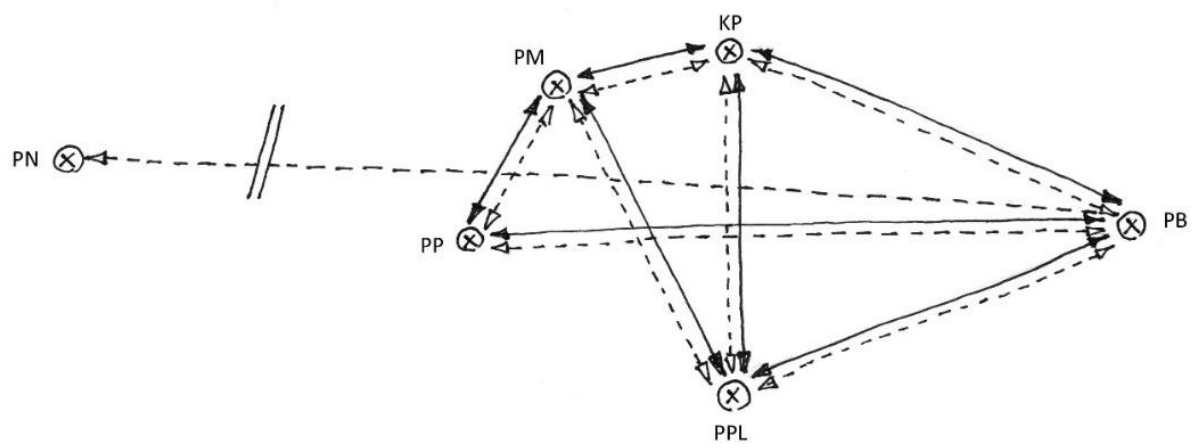

Figure 10. Diagramme des relations entre divers points de vue du Jardin de la Politique des Simples (source : F. Li). PPL : Pavillon-des-parfums-lointains, PB : Pavillon-bambou, KP : Kiosque-du-parfum-de-laneige-et-des-nuages-bénéfiques, PP : Porte-pavillon-d'un-pavillon-au-charme-exceptionnel, PM : Pavillond'où-l'on-voit-les-montagnes, PN : Pagode-du-nord

Pour autant, l'emprunt de scène et la conception de la perception de ce jardin ne peut se résumer à son aspect visuel. En effet, le jardin sollicite nos sens au-delà d'une simple perception visuelle : l'ouïe, l'odorat.

\section{Et les autres sens ? : l'ouïe et l'odorat}

L'emprunt de scène fait également appel à nos autres sens comme l'évoque Ji Cheng dans son traité à son propos : «En été. [...] Lotus émergeant de l'onde, fraîcheur éclatante; des gouttes de pluie légères sur les feuilles de bambou, sonorité de jade. Contempler un bosquet de bambous au creux d'un torrent, observer les poissons depuis une digue [...]. En automne. Le vêtement en ramie ne protège plus contre la fraîcheur récente, alors que l'étang de lotus en retient encore la fragrance. » (Ji 1997, 285-87)

Dans le cadre du jardin de la Politique des Simples, prenons l'exemple du patio jouxtant le Pavillon-à-l'écoute-de-la-pluie (PE) (figure 11). Ce patio est planté de bambous qui, lorsqu'il pleut, produisent le son affectionné par Ji Cheng. L'architecturologie ne dispose pas apparemment d'échelle pour décrire la conception de ce dispositif sonore, faisant référence à l'ouïe. Serait-il 
possible d'ajouter à son système conceptuel, une échelle sonore ? Pour cela il faudrait la distinguer de celles déjà existantes susceptibles d'expliquer ce phénomène.

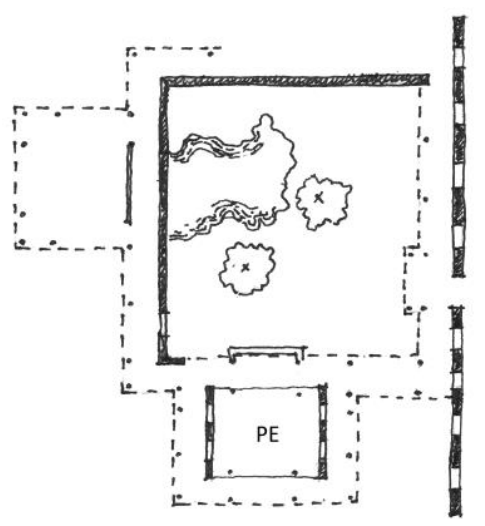

Figure 11. Plan du patio près du Pavillon-à-l'écoute-de-la-pluie (Liu 2005, 315)

L'échelle fonctionnelle consiste en l'attribution de mesures à un espace selon un usage. Mais contrairement à cet usage, la sollicitation de l'ouïe ne répond pas à une action intentionnelle de l'utilisateur. L'échelle acoustique récemment proposée par Dominique Raynaud s'apparente à une première conceptualisation du phénomène de conception qui nous intéresse. Il désigne par cette échelle le fait de « donner forme ou mesure à un édifice afin de maitriser l'incidence des sons sur l'homme » (Raynaud 2017). Dans ce cas, nous pouvons nous interroger sur sa distinction avec l'échelle technique attribuant des mesures selon des considérations d'ordre mécanique et physique.

Par souci de clarté, le terme de sonore sera employé afin d'éviter la confusion avec la technicité à laquelle renvoie la discipline acoustique. De plus Dominique Raynaud ne fait pas, la distinction entre " entendre » et "être entendu » à l'image des échelles optique et de visibilité. Sous ses conditions, nous pourrions parler d'échelle sonore lorsqu'il s'agit d'attribuer des mesures à un objet destiné à être entendu en tenant compte d'un point d'audition. Il faudrait alors imaginer l'échelle d'audibilité décrivant la constitution d'un point d'audition en tenant compte d'une source sonore (figure 12).

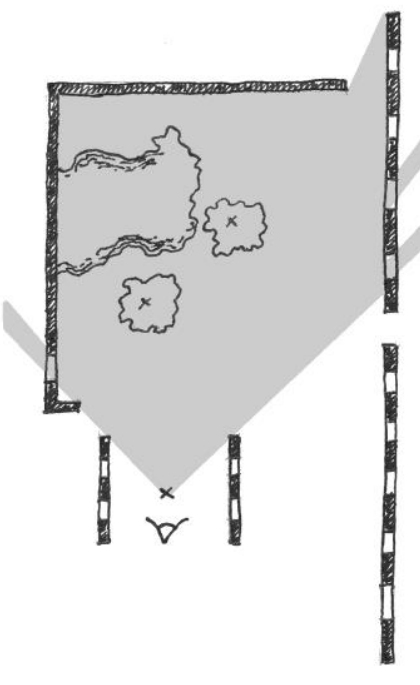

échelle de visibilité

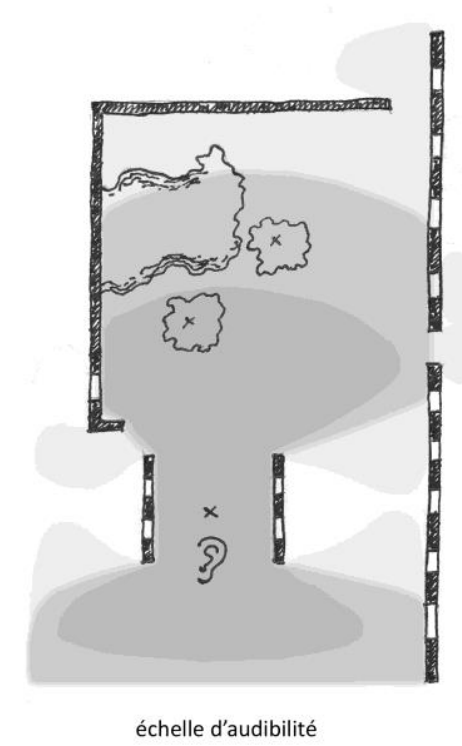

échelle d’audibilité

Figure 12. Schéma analogique entre échelle de visibilité et d'audibilité (source : F. Li) 
Une démarche similaire effectuée sur l'odorat, permettrait d'expliquer la conception relative au parfum des lotus par exemple ; débouchant sur des échelles d'olfaction et de perception olfactive. Afin d'éviter une extension du nombre d'échelles, il est envisageable de les regrouper sous deux échelles plus englobantes (figure 13).

\begin{tabular}{|l|l|}
\hline \multicolumn{2}{|c|}{ Échelle de perceptibilité } \\
\hline Définition & $\begin{array}{l}\text { Constitution d'un point de perception dans l'espace de manière à } \\
\text { percevoir un objet donné }\end{array}$ \\
\hline Sous-échelles & $\begin{array}{l}\text { - échelle de visibilité (vue) } \\
\text { - échelle d'audibilité (ouie) } \\
\text { - échelle d'olfactibilité (odorat) }\end{array}$ \\
\hline Définition & $\begin{array}{l}\text { Tenir compte d'un point de perception pour situer un objet dans } \\
\text { l'espace de perception }\end{array}$ \\
\hline Sous-échelles & $\begin{array}{l}\text { - échelle optique (vue) } \\
\text { - échelle sonore (ouie) } \\
\text { - échelle d'olfaction (odorat) }\end{array}$ \\
\hline
\end{tabular}

Tableau 2. Proposition d'une nouvelle catégorisation des échelles dites « sensorielles »

Ces exemples illustrent la création de nouveaux outils architecturologiques pour décrire la conception des scènes du jardin de la Politique des Simples. Toutefois, ces scènes ne se limitent pas à une conception de leur perception. Dans la scène principale, la vue sur des «montagnes artificielles est ainsi mobilisée dans le but d'évoquer des références d'ordre géographique (le mont Xian, la nature sauvage), socio-culturel (expressions picturales, poétiques du shanshui ${ }^{8}$ ) et symbolique (l'érémitisme) (Escande 2005, p. 193 et 241).

\section{Projet de Clichy-Batignolles}

Le secteur d'aménagement de Clichy-Batignolles (Paris XVIIème arr.) est porteur d'objectifs énergétiques très ambitieux. Se proclamant comme une référence du développement urbain durable (Rougé 2015), cette opération témoigne d'un urbanisme contemporain structuré autour du parc Martin Luther-King de 10 ha. La paysagiste Jacqueline Osty déclare que « le parc Martin Luther-King épouse la ville et inversement. On emprunte le parc comme on emprunte une rue »(Osty 2013). Ainsi, il est intéressant de se pencher sur la relation que les concepteurs établissent entre le parc et le bâti au sein du quartier.

\section{La conception du parc Martin Luther-King}

L'acte fondateur du projet de l'écoquartier de Clichy-Batignolles repose sur la création d'un grand parc urbain dédié aux loisirs et à la détente au milieu du site autour duquel se développent les bâtiments. Ce parc se veut un espace de rassemblement et de réunion des quartiers alentours (Dossier de réponse, 2009, p. 17) : ses limites s'étendent aux immeubles limitrophes et sont visuellement fluides.

\footnotetext{
${ }^{8}$ Traduit littéralement « montagnes et eaux », terme signifiant paysage. 
Cette insertion du parc dans les îlots était pensée par les concepteurs du quartier François Grether, Jacqueline Osty et associés ${ }^{9}$ par l'idée « d'ouvrir des vues directes et étendues depuis la profondeur des îlots » mais également « d'agrandir les dimensions par des vues vers les espaces intérieurs des îlots » (Cahier des Prescriptions Architecturales et Paysagères, cité in Dossier de réponse, 2009, p. 33). Les nombreux cheminements, qui le traversent en prolongeant la trame urbaine, contribuent ainsi à la continuité et à l'insertion du paysage environnant à l'intérieur du parc (cf. Figure 14).
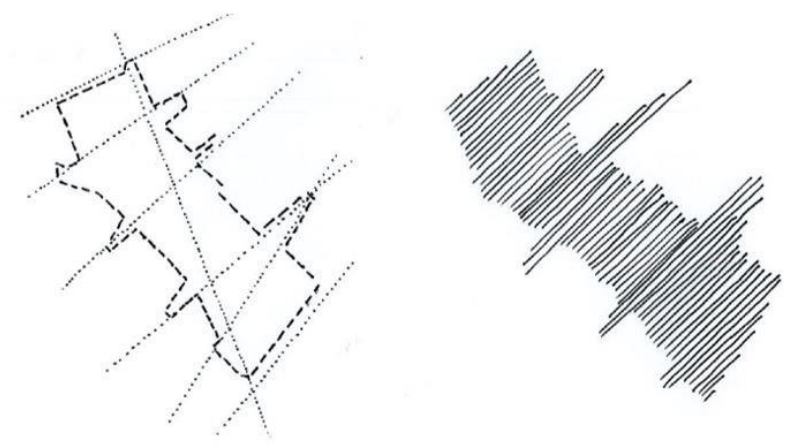

Figure 14. Le parc traversé par des axes (a) et conçu comme un espace de liaison où la nature se prolonge aux cœurs d'ilots et sur l'espace public (b) (source : M. Apostolou)

Cette idée d'extension du parc vers la ville renvoie « à l'image de la ville contemporaine et durable, au Paris du 21 ${ }^{\text {ème }}$ siècle »(Dossier de réponse, 2009, p. 29). Plutôt que les modèles haussmanniens du $19^{\text {ème }}$ siècle qui isolent une nature domestiquée à l'image du parallélépipède du square des Batignolles, la paysagiste a créé « des entrées végétalisées pour faire une « couture » avec les quartiers environnants » (Osty 2013).

Ce choix de conception pourrait être architecturologiquement décrit par la mise en place d'une échelle de visibilité de pertinence globale, qui fabrique une façon de voir la ville sans discontinuité depuis le parc. De plus, les limites du parc sont brouillées d'abord visuellement : de multiples points de vue sont constitués en profondeur dans les cœurs des îlots sur le parc mais aussi sur les parties communes végétalisées des immeubles. Il s'agit d'une échelle optique qui est aussi accentuée par une géométrique : le contour général du parc ne dessine pas une forme clairement identifiable. La forme dentelée des entrées végétalisés génère des excroissances du parc qui s'insèrent au milieu du bâti.

Les concepteurs disent également avoir pris en compte l'entourage du parc. Ainsi, les axes allées et cheminements - le traversent en prolongeant la trame urbaine prévue à l'intérieur. Des bassins biotopes ont été créés dans un souci d'économies en irrigation et de développement de la biodiversité. Enfin, les traces du passé ferroviaire du site sont dites intégrées au parc de manière à mettre en scène leur histoire : les lignes des rails portants la mémoire se remodèlent en s'insérant au nouveau paysage qui émerge en particulier dans le « jardin du rail ».

Ces aspects de la conception interne du parc peuvent être architecturologiquement interprétés. La continuité spatiale est induite relativement à des éléments appartenant du voisinage et relèvent ainsi de l'échelle de voisinage qui soulève la question de ses propres limites. Le découpage de l'espace par la présence des bassins relève d'une échelle économique qui concerne ici le recyclage et la

\footnotetext{
${ }^{9}$ Le parc Martin Luther King est réalisé et entretenu par la Direction des Espaces Verts et de l'Environnement de la Ville de Paris, avec Atelier Jacqueline Osty et associés (paysagistes), Atelier François Grether (architectes urbanistes), OGI (Bureau d'études) et Concepto (concepteurs lumière).
} 
récupération des eaux à des fins écologiques et environnementales. La taille du bassin et son positionnement central sont induits par la pertinence de l'économie en eau et de sa gestion. L'expression « jardin du rail » (petit jardin thématique) agit comme une échelle sémantique qui découpe un espace dans lequel une échelle symbolique devient dominante : cette partie est conçue en référence à la mémoire du site. « Nous avons pris le parti d'intégrer cet aspect au paysage. Au lieu d'en faire une contrainte, nous avons cultivé l'aspect industriel du site » (Osty in Sérès, 2011). Ainsi, la taille et l'orientation des lignes ferroviaires sont conservées comme structure des allées. Cette échelle est relayée par une échelle optique qui conduit à laisser les rails visibles et à réutiliser les pavés de l'ancien site ferroviaire.

\section{La conception du bâti par rapport au parc}

Cette forte volonté globale de prolonger le parc dans les îlots et les espaces environnants impacte non seulement la conception du parc mais aussi celle des bâtiments autour de lui. Dans cette partie on se focalisera sur la conception du bâti par rapport au parc à travers la mise en place des interactions visuelles de « voir et être vu » réalisées dans plusieurs lots (cf. Figure 15). Via l'échelle de visibilité, les espaces de végétation à l'intérieur des lots sont dessinés pour donner à voir la végétation du parc. Cette échelle est mise en place soit par un découpage de l'espace bâti par la végétation (lot o4b et e2) ; soit par un découpage du lot eu égard aux chemins visuels provenant du parc ainsi qu'au positionnement et à l'orientation du bâti en manière qui les poursuit (o8, o6a); soit par le placement des limites végétalisés autour des lots (o6a) ou à leur intérieur dans des niveaux différents (o6b) qui pourrait renvoyer à une échelle parcellaire degré zéro.
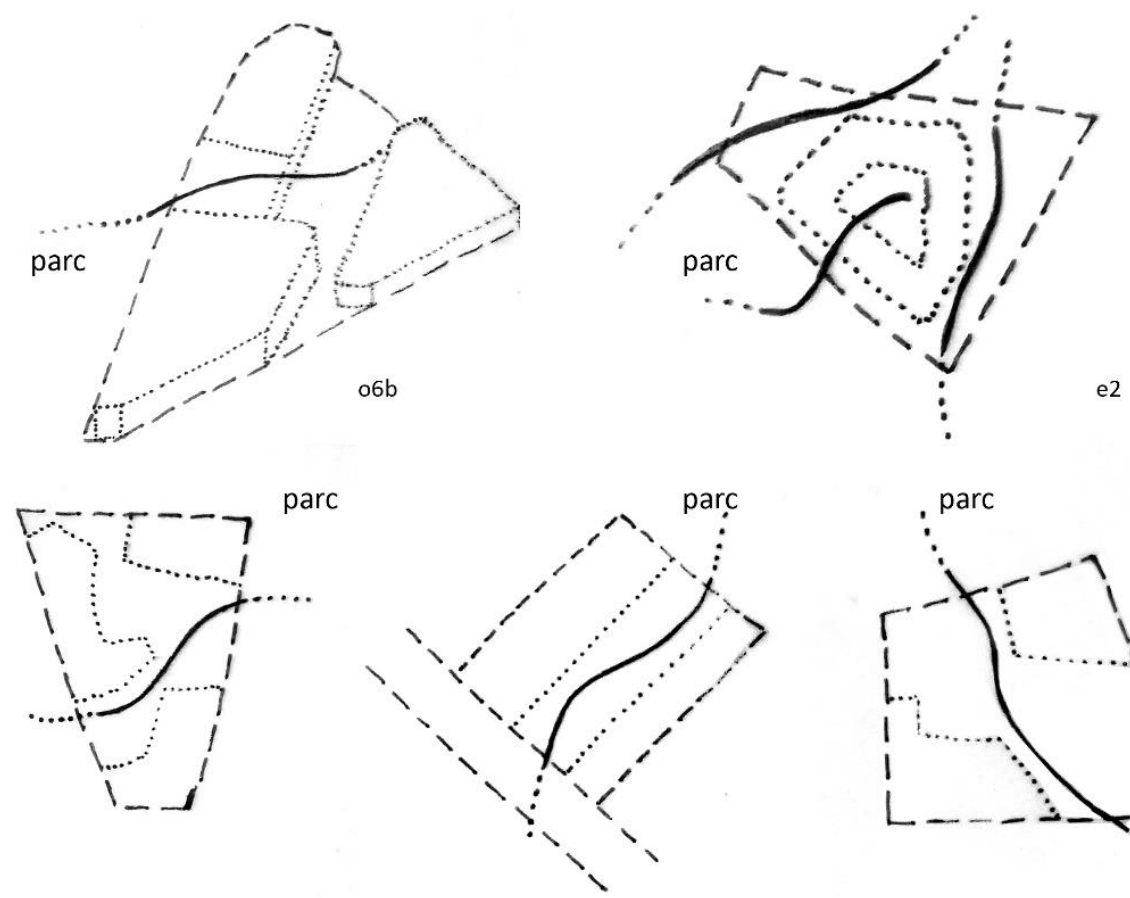

08

04

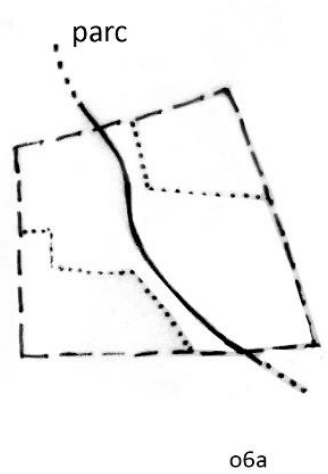

Figure 15. Prolongement du parc dans des îlots (source : M. Apostolou).

Le parc est prolongé de diverses manières dans les îlots : un potager aromatique en toiture à $\mathrm{R}+1$ avec vue sur le parc et un jardin collectif au cœur d'îlot (o6b) ; aménagement des chemins visuels qui suivent le parc à l'intérieur de l'îlot et l'amènent à la rue $(08)$; un jardin découpe la parcelle en créant un prolongement du paysage et une porosité entre la rue et le parc (o4b) ; découpage de l'espace et faille visuelle pour la perception du paysage de parc depuis la rue - placement d'un banc végétal au long du mur pour poursuivre le paysage végétalisé du parc (o6a) ; positionnement du bâtiment pour inviter le parc et une cour intérieure liée visuellement au parc par la transparence du $\operatorname{rdc}(\mathrm{e} 2)$. 
Ces exemples témoignent de diverses manières de la mise en place d'une échelle de visibilité dans la conception de cet espace. Le paysage urbain se veut ici aménagé par la nature, qui, comme une « percée visuelle » s'infiltre sur tous les axes du quartier (arbres-de l'espace public, murs végétalisés, terrasses plantés, façades, jardins à l'intérieur des îlots) en créant des prolongements visuels en lien avec le parc. Ces relations poreuses entre extérieur et intérieur se manifestent par des recherches de transparences et de perspectives qui s'articulent autour du «voir et être vu ». Si la mise en scène du paysage passe ici par des relations visuelles, le quartier veut se proclamer comme une ville paysage où la fusion des objets artificiels spatiaux se construit au-delà de l'usage de la nature comme élément décorateur.

\section{Conclusion de l'expérience}

Cette analyse montre comment l'architecturologie est susceptible d'interpréter différents cas de conception paysagère. Le fait que des échelles architecturologiques peuvent être repérées dans l'analyse, montre que la conception des trois paysages envisagés procède par attribution de mesures. Les concepteurs de paysages conçoivent leur parcs et jardins en leur attribuant des mesures. Les modalités de ces mesures sont diverses, néanmoins on peut remarquer que l'échelle optique intervient dans chacun des cas d'étude. Bernard Lassus détermine la pente et la hauteur de la dalle couvrant La Villette de manière à simuler un "horizon". Dans les jardins de Suzhou, les maîtres concepteurs positionnent des pavillons de manière à créer des scènes vues depuis des points clés de parcours. La forme irrégulière du parc de Clichy-Batignolles s'insère dans le bâti, multipliant ainsi les relations visuelles et donc la présence de la nature en ville.

Mais les cas présentent aussi des différences notables : La Villette semble être un parc d'architecte tant par les réponses que par la commande. Les jardins de Suzhou ont l'esthétique traditionnelle inspirée d'une spiritualité orientale. Clichy-Batignolles apparaît comme un quartier urbain qui veut renouveler le rapport des citadins à la nature. L'interprétation architecturologique observe en effet des échelles différentes ayant des rôles clés : l'échelle géométrique semble prégnante chez les architectes Bernard Tschumi et Rem Koolhaas. Des échelles de perception auditive et olfactive caractériseraient le jardin de la Politique des Simples. L'échelle économique intervient dans le parc Martin Luther-King inspiré par un espace de référence écologique ${ }^{10}$. De plus, la même échelle optique, repérée dans les trois cas, opère de trois manières différentes. Ces différentes modalités de mise en œuvre ${ }^{11}$ de l'échelle peuvent être architecturologiquement décrites notamment au niveau des références à partir desquelles elles opèrent : la « vue » n'a pas le même sens pour Bernard Lassus, les jardiniers chinois du XVII ${ }^{\text {ème }}$ ou pour Jacqueline Osty.

Ainsi nous pouvons conclure cette expérience en proposant que le repérage de ces similitudes et différences par le moyen de l'interprétation architecturologique témoigne d'un apport possible pour l'analyse de la conception paysagère. L'architecturologie, permet une description procédurale de la conception, en cela elle opère un déplacement des paysages réalisés vers leur mode de réalisation. Elle n'exclut pas la perception, mais l'intègre en tant qu'un des points de vue actifs de la conception. Néanmoins, nous pouvons penser à raison que l'architecturologie n'épuise pas la description du paysage. En particulier les différences entre nos trois cas relèvent notamment de questions culturelles qu'une autre théorie, la mésologie, semble pouvoir saisir et vers laquelle nous souhaitons maintenant nous tourner.

10 Cette interprétation est bien entendue relative à l'aspect partiel de l'étude des cas. Elle serait à étayer par une analyse plus exhaustive.

11 L'architecturologie désigne le produit d'une échelle comme étant un scalème, il y a une infinité de scalèmes pour une même échelle. 


\section{Architecturologie et mésologie ?}

En premier lieu, rappelons que la mésologie se définit selon Augustin Berque comme une science des milieux étudiant la relation entre êtres humains et environnement (Berque 1986, 15). La notion de milieu permet d'aller au-delà de la distinction entre sujet et objet, entre culture et nature. Cette dualité est souvent convenue en Occident ; l'être humain doté de pensée et de culture se détacherait de son environnement naturel concret. Au contraire, la mésologie considère la nature comme empreinte d'une expression culturelle de même que la culture puise ses racines dans la nature. La trajectivité exprime le fait qu'une réalité, un paysage n'est ni strictement objectif, ni subjectif, mais trajectif (Berque 1986, 94 et 164). Ainsi, un objet, un paysage physiquement matérialisé est toujours saisi dans une interprétation par l'être, il est saisi « en tant que » quelque chose.

À partir de cette réflexion sur les milieux, nous posons une complémentarité avec l'architecturologie pour affiner le travail précédemment effectué. Nos trois cas peuvent être alors plus particulièrement observés sous le prisme de cette trajectivité. Pour comparer, restreignons-nous aux échelles optiques et économiques mobilisées dans les cas :

Dans le parc de La Villette de Bernard Lassus, l'échelle optique est utilisée comme un moyen de prendre conscience de l'idée de géographie à travers une pente topographique. Elle cherche à aller au-delà de l'histoire récente afin de révéler sa grande histoire, son territoire. Économiquement, ce projet représente délibérément un budget conséquent qui est investi pour la collectivité.

Dans la conception du jardin de la Politique des Simples, l'échelle optique s'exprime comme un moyen de lier l'homme à une géographie sensible, un moyen d'être en prise avec la nature et de catalyser la méditation du visiteur. Elle reflète également une connexion forte avec l'histoire culturelle chinoise et ses arts. L'échelle économique s'illustre notamment lors de la création de « montagnes » artificielles, qui implique un budget conséquent pour des individus privés.

Quant au quartier de Clichy-Batignolles, l'échelle optique est employée pour lier les différents îlots au parc à travers une continuité végétale. De plus, les concepteurs font ici référence à une géographie entendue comme naturelle ou « libre, » qui suggère des activités de loisirs. Sur le plan économique c'est une économie de moyens qui est recherchée et qui se traduit par une conservation de la topographie.

Au premier abord, chacun de ces paysages sollicite la vue. Cette vue, à savoir le fait de percevoir les rayons lumineux de longueurs d'ondes variées du spectre visible, est l'objet réel en mésologie. Il en est de même pour l'économie qui possède une réalité à travers le coût de réalisation de ces projets. Pour autant il ne s'agit ici que d'une description objective, partielle, du milieu qui est trajectif.

En effet, nous remarquons à travers l'échelle optique qu'il y a une trajection de ce qui est vu en tant que géographie naturelle. Chez Bernard Lassus, cette géographie est perçue en tant que concept métaphysique. Du point de vue des maîtres jardiniers chinois, la géographie est un concept occidental et la nature est appréciée plutôt en tant que scène ; cette dernière étant elle-même conçue comme un moyen de favoriser la méditation. Enfin, au XXI ${ }^{\text {ème }}$ siècle la nature apparaît comme quelque chose avec laquelle les citadins cherchent à reprendre contact. Ainsi, la nature pensée par Jacqueline Osty comme « libre »s'oppose à celle de l'urbanité haussmannienne ; elle encourage des activités de relaxation. Dans les deux cas sus-cités, la relaxation à Clichy-Batignolles et la méditation à Suzhou diffèrent, du fait de leurs milieux distincts. D'un côté il s'agit d'une pratique occasionnelle accessible à tous dans un mode de vie urbain. Au sein du jardin traditionnel chinois, elle correspond à une forme d'érémitisme lié à une pratique régulière seulement ouverte aux lettrés, soit une classe sociale éduquée. 
La prise en compte économique dans la conception paysagère peut, quant à elle, être comprise comme la trajection d'un coût, alloué en tant que bien collectif du côté des projets contemporains, en tant que bien privé dans les jardins de Suzhou. A nouveau, ces biens nécessitent d'être saisis dans leur milieu social. A Suzhou, la réalisation de ses jardins privés correspond à une période prospère dans une région en plein essor économique. De même, les investissements importants accordés au projet du parc de la Villette sont cohérents avec un gouvernement socialiste de " grands projets ». $\mathrm{Au}$ contraire, le parc Martin Luther-King s'inscrit, lui, dans un contexte économique moins favorable concomitant à la montée du discours écologique. Un projet de dalle à la manière de Bernard Lassus aurait été culturellement déconnecté du contexte.

Ces exemples illustrent que la mésologie permet dans une certaine mesure d'affiner l'analyse de la conception paysagère effectuée par le biais des échelles architecturologiques. La vue et l'économie ont leurs propres trajections au milieu de chacun des projets, situé tout autant spatialement que temporellement. Cette étude ne demande qu'à être étayée par d'autres cas, qui permettront de mieux comprendre l'apport du milieu pour la prise en compte mutuelle de la nature et de la culture dans la conception du paysage.

\section{Conclusion}

Cette exploration a permis d'envisager que le rapport de l'architecturologie à une spécificité architecturale reposait in fine sur l'habitude des chercheurs architecturologues plutôt que sur la structure de la théorie elle-même. Les postulats que sont la mesure et la conception sont alors susceptibles de s'appliquer à la conception paysagère de la même manière qu'ils s'appliquent à la conception architecturale. Une fois posé de manière théorique, cela a été envisagé à travers plusieurs cas d'études de paysages. Nous avons montré que l'architecturologie ouvre une piste de travail permettant de comparer trois cas de paysages grâce à un cadre d'analyse commun qui permet de repérer ce qui leur est commun et ce qui les distingue. D’autres cas auraient pu être traités pour renforcer ce constat. Cela ne fait pas de l'architecturologie un outil absolu qui épuiserait toute la connaissance du paysage, pas plus qu'elle n'épuise la connaissance de l'architecture. Il est alors possible d'envisager comment elle s'articule avec d'autres théorisations déjà développées dans les études sur le paysage. Nous avons ainsi indiqué l'opportunité d'un croisement avec la mésologie d'Augustin Berque. Une connaissance plus fine des rapports nature-culture permet en effet d'enrichir la compréhension des différences entre les conceptions paysagères. Elle met en lumière la manière dont les références mobilisées dans les projets sont déterminées par un contexte culturel qui influencent les concepteurs sans qu'ils en soient toujours conscients. L'architecturologie de son côté permet de comprendre le rôle actif des concepteurs qui mobilisent ces références dans la conception d'espaces paysagers.

On pourra toujours arguer que la lecture architecturologique transforme les paysages en les approchant comme des architectures. On pourrait penser que l'essence végétale par exemple échapperait à la focalisation sur la mesure et donc à l'architecturologie. Pourtant, il faut considérer une perspective bachelardienne pour laquelle l'abstraction est un gain dans les sciences. La mesure peut être alors comprise comme une notion abstraite. Plutôt que la penser uniquement en mètres, mètres carrés ou cubes, nous avions déjà décrit le choix de matières comme des attributions de mesures (Vitalis et Guéna 2016). Ne peut-on pas alors, considérer le choix d'une essence végétale comme une attribution de mesure au sens qu'en donne l'architecturologie ? Décrire architecturologiquement un parc, ne conduit pas selon nous à penser le parc comme une architecture, simplement à proposer une description abstraite, ou non-substantialiste de sa conception qui n'en exclut pas d'autres. 


\section{Bibliographie}

BERQUE A., Le sauvage et l'artifice : les Japonais devant la nature, Gallimard, Paris, 1986.

BERQUe A., Écoumène. Introduction à l'étude des milieux humains, Belin, Paris, 2010.

Blanche R., L'axiomatique. Quadrige. PUF, Paris, (1955) 1990.

Boudon P., «Paysage de l'architecture. Architecture du paysage ». Les Annales de la Recherche Urbaine 18 (1) : p. 142-55. 1983.

Boudon P., «Jeu de l'oie, jeu de l'échelle» dans HAMON P., Littérature \& architecture, Presses universitaires de Rennes II, Rennes, 1988.

Boudon P., «Architecturologie et poïtique », Revue Luxembourgeoise de Littérature Générale et Comparée - Littérature et architecture Colloque du 25anniversaire, p. 10-22. 2004.

Chomarat-Ruiz C., Précis de paysagétique, Presses universitaires de Valenciennes, Valenciennes, 2014.

EPPV. «Procès-verbal du jury international pour le parc de La Villette », dans Conférence de presse, Paris, 1982.

ESCANDE Y., Montagnes et eaux : la culture du Shanshui, Hermann, Paris, 2005.

Fung S., «Here and there in Yuan ye », Studies in the History of Gardens \& Designed Landscapes 19 (1), p. 36-45, 1999.

Ji C., Yuanye le traité du jardin (1634) : Traduit du Chinois par C. B. CHIU, Les Éditions de L'Imprimeur, Paris, 1997.

JIE L., et ZHENG X., "Influences of Literati painting on garden-making: a comparative study of Yuan ye (The Craft of Gardens) and Fu sheng liu ji (Six Chapters of a Floating Life) », Studies in the History of Gardens \& Designed Landscapes 37 (4), p. 336-41, 2017.

KoOlHAAs R., «Une Arcadie synthétique », l'Architecture d'aujourd'hui, nº 227 (juin), p. 91-99, 1983.

KoolhaAs R., «Parc de la Villette. Paris, 1982-1983», dans LuCAN J., Rem Koolhaas. Pour une culture de la congestion, p. 56-65, Electa Moniteur, Paris, 1990.

LASsus B., «Profondeurs », Les Annales de la Recherche Urbaine, $\mathrm{n}^{\mathrm{o}}$ 18, p. 128-41, 1983.

Lassus B., The Landscape Approach, University of Pennsylvania Press, Philadelphie, 1998.

LIU D., Classical gardens of Suzhou, Zhongguo jian zhu gong ye chu ban she, Beijing, 2005.

ORLANDINI A., La Villette 1971-1995 : Histoires de projets, Somogy, Paris, (1999) 2003.

OSTY, J., «Voir et vivre la nature au cœur de la ville», URL : https://archive-clichybatignolles.parisetmetropole-amenagement.fr/voir-et-vivre-la-nature-au-coeur-de-la-ville-460.html. 2013.

RAYNAUD D., «Échelles et raisons d'agir dans la conception architecturale », Sciences du Design $\mathrm{n}^{\circ} 5$ (1), p. 131-44, 2017.

RougÉ N., « Clichy-Batignolles: Where urban planning meets the climate », OECD Observer, 2015.

SEMAVIP, «Ecoquartier Clichy Batignolles, dossier de réponse, Paris 17e », Mairie de Paris, Semavip, https://archive-clichy-batignolles.parisetmetropole-

amenagement.fr/sites/default/files/clichybatignolles_23-3-9_0.pdf. 2009.

SERES A., «Une voie ferrée va balafrer le nouveau parc des Batignolles », Le Figaro (blog), 2011. https://www.lefigaro.fr/actualite-france/2011/10/17/01016-20111017ARTFIG00695-une-voie-ferree-vabalafrer-le-nouveau-parc-des-batignolles.php. 2011.

Tschumi B., Le parc des folies, les folies du parc de la Villette à Paris, projet 749 - rendu du concours : cahier: Archives Nationales, Pierrefitte-sur-Seine, 1982.

Tschumi B., «Bernard Tschumi le parc de La Villette. Entretien avec l'architecte (entretien avec Jacques Lucan) », $A M C, \mathrm{n}^{\circ}$ 6, p. 32-45, 1984. 
Tschumi B., Cinégramme Folie - Le parc de La Villette, Princeton architectural press, Princeton, 1989.

Tschumi B., éd. Tschumi Parc de la Villette, Artifice Books on Architecture, Londres, 2014.

Vitalis L., et Guena F., «La matière, obstacle épistémologique? » Le Philotope, $\mathrm{n}^{\mathrm{o}} 12 \mathrm{MaT}(\mathrm{i}) \operatorname{erre}(\mathrm{s})$ (décembre), p. 89-95, 2016. 